\title{
A Review of the Potential Utility of Mycophenolate Mofetil as a Cancer Therapeutic
}

\author{
Nazanin Majd, ${ }^{1}$ Kazutaka Sumita, ${ }^{2,3}$ Hirofumi Yoshino, ${ }^{2}$ \\ Dillon Chen, ${ }^{2}$ Jumpei Terakawa, ${ }^{4}$ Takiko Daikoku, ${ }^{4}$ Satoshi Kofuji, ${ }^{2}$ \\ Richard Curry, ${ }^{1,2}$ Trisha M. Wise-Draper, ${ }^{2}$ Ronald E. Warnick, ${ }^{3}$ \\ Jessica Guarnaschelli, ${ }^{5}$ and Atsuo T. Sasaki ${ }^{2,3,6}$ \\ ${ }^{1}$ Department of Neurology, University of Cincinnati Medical Center, 260 Stetson Street, Suite 2300, Cincinnati, OH 45267-0525, USA \\ ${ }^{2}$ Division of Hematology-Oncology, Department of Internal Medicine, UC Cancer Institute, 3125 Eden Avenue Room 2112, \\ Mail Loc-0562, Cincinnati, OH 45221, USA \\ ${ }^{3}$ Department of Neurosurgery, UC Brain Tumor Center, 260 Stetson Street, Mail Loc-0515, Cincinnati, OH 45221, USA \\ ${ }^{4}$ Department of Reproductive Medicine, Cincinnati Children's Hospital Medical Center, Mail Loc-0054, Cincinnati, OH 45221, USA \\ ${ }^{5}$ Department of Radiation Oncology, Trihealth Cancer Institute, 4415 Aicholtz Road, Cincinnati, OH 45245, USA \\ ${ }^{6}$ Vontz Center for Molecular Studies, 3125 Eden Avenue Room 2112, Cincinnati, OH 45221, USA
}

Correspondence should be addressed to Atsuo T. Sasaki; atsuo.sasaki@uc.edu

Received 20 January 2014; Revised 18 March 2014; Accepted 21 March 2014; Published 7 July 2014

Academic Editor: Takahiro Yamauchi

Copyright (c) 2014 Nazanin Majd et al. This is an open access article distributed under the Creative Commons Attribution License, which permits unrestricted use, distribution, and reproduction in any medium, provided the original work is properly cited.

Tumor cells adapt to their high metabolic state by increasing energy production. To this end, current efforts in molecular cancer therapeutics have been focused on signaling pathways that modulate cellular metabolism. However, targeting such signaling pathways is challenging due to heterogeneity of tumors and recurrent oncogenic mutations. A critical need remains to develop antitumor drugs that target tumor specific pathways. Here, we discuss an energy metabolic pathway that is preferentially activated in several cancers as a potential target for molecular cancer therapy. In vitro studies have revealed that many cancer cells synthesize guanosine triphosphate (GTP), via the de novo purine nucleotide synthesis pathway by upregulating the rate limiting enzyme of this pathway, inosine monophosphate dehydrogenase (IMPDH). Non-proliferating cells use an alternative purine nucleotide synthesis pathway, the salvage pathway, to synthesize GTP. These observations pose IMPDH as a potential target to suppress tumor cell growth. The IMPDH inhibitor, mycophenolate mofetil (MMF), is an FDA-approved immunosuppressive drug. Accumulating evidence shows that, in addition to its immunosuppressive effects, MMF also has antitumor effects via IMPDH inhibition in vitro and in vivo. Here, we review the literature on IMPDH as related to tumorigenesis and the use of MMF as a potential antitumor drug.

\section{Introduction}

Metabolic regulation in tumors is different from resting adult tissues. Tumor cells increase synthesis of proteins, lipids, and nucleotides to adapt to their rapid proliferation. Nearly, a century ago, Dr. Otto Warburg first described the altered metabolism of cancer cells, known as the "Warburg effect." The Warburg effect is the observation that the energy needed by tumor cells is mainly produced through high rates of glycolysis followed by lactic acid fermentation in the cytosol, as opposed to the low rate of glycolysis followed by pyruvate oxidation in the mitochondria of nonmalignant cells. The relationship between tumor and metabolism has just recently been a major focus of cancer biology. Growing evidence supports a pivotal role for oncogenic mutations and the resultant altered signaling pathways that dramatically change cell metabolism. For example, phosphatidylinositol 3kinase (PI3K) and mammalian target of rapamycin (mTOR) signaling pathways are established oncogenic drivers in a variety of cancers [1]. These signaling pathways lead to increased glucose uptake and many anabolic processes, such as lipid biosynthesis and protein and nucleotide synthesis [2]. Potent inhibitors of PI3K and mTOR have been designed, and several of them are currently in clinical trials targeting 


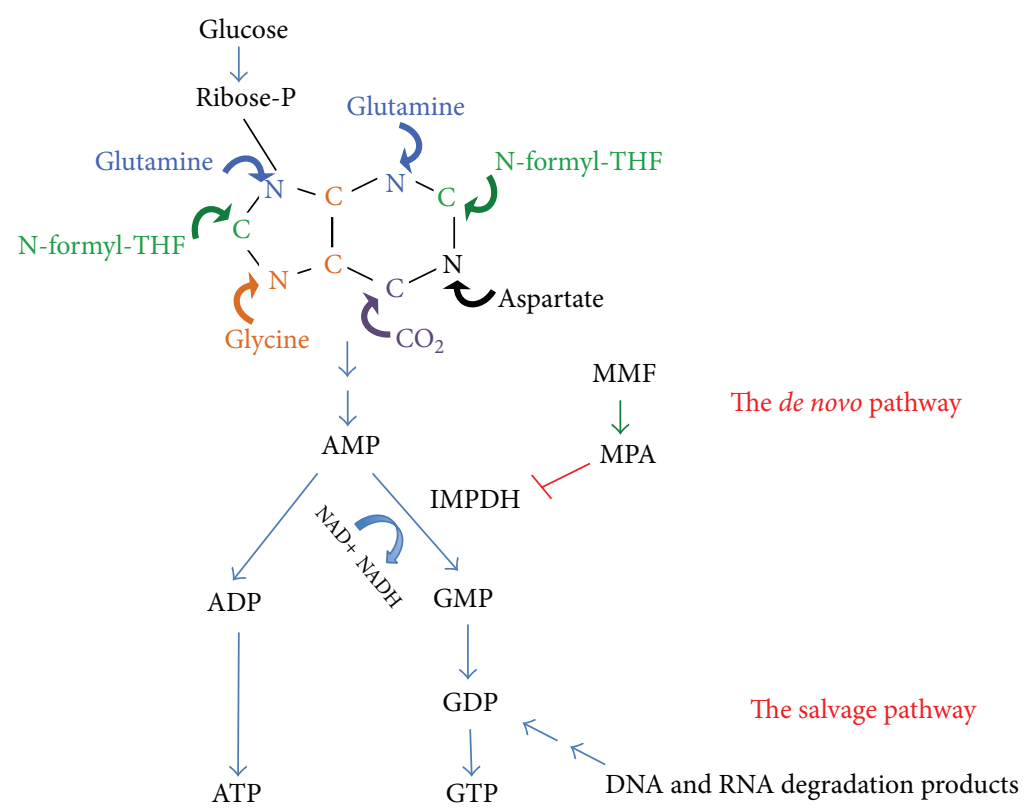

FIGURE 1: Purine nucleotide synthesis pathways. IMP: inosine monophosphate and IMPDH: inosine monophosphate dehydrogenase. XMP: xanthine monophosphate, AMP: adenosine monophosphate, and GMP: guanine monophosphate. GTP: guanosine triphosphate, ATP: adenosine triphosphate, and NAD: nicotinamide adenine dinucleotide. MMF: mycophenolate mofetil, and MPA: mycophenolic acid.

various cancers [3]. However, acquired resistance to these inhibitors via mutation of specific binding proteins or recurrent alteration of signaling pathways is an emerging problem [4]. Therefore, alternative approaches to target tumor cell specific metabolic pathways need to be considered.

In this review, we focus on metabolic pathways that are specifically upregulated in tumors. The aim is to target key enzymes in such pathways to suppress cell proliferation or induce differentiation of cancer cells. We focus on one of the major energy currency molecules, GTP. In addition to its well-known role as a building block of $m$ RNA and DNA, GTP has distinctive roles as the cellular energy source. GTP is a critical energy molecule for protein synthesis and signaling via GTP-binding proteins. Most importantly, GTP levels are more elevated in several cancers than that of ATP [5] making the GTP synthesis pathway of particular interest in cancer biology. GTP upregulation may reflect the increased need for protein synthesis in tumor cells as opposed to resting, noncancerous cells. The knowledge of these two important aspects of GTP raises the following questions.

\section{How do cancer cells upregulate GTP synthesis?}

Is GTP upregulation important for tumor cell growth?

Does targeting GTP synthesis pathway result in antitumorigenesis?

To address these questions, we review the current literature on the role of the rate-limiting enzyme of the GTP nucleotide synthesis pathway, inosine monophosphate dehydrogenase (IMPDH), in various malignancies. We also review the body of work that has explored the role of FDA-approved IMPDH inhibitor, mycophenolate mofetil (MMF), as an antitumor drug.

\section{How Do Cancer Cells Upregulate GTP Synthesis?}

2.1. Various Tumors Utilize the De Novo Purine Nucleotide Synthesis Pathway to Synthesize GTP. Cells use two distinct pathways to generate ATP and GTP: the "salvage pathway" and the "de novo purine nucleotide synthesis pathway." The salvage pathway is an energy efficient pathway that utilizes assembled parts of nucleotides formed during degradation of DNA and RNA to make ATP and GTP. The de novo purine nucleotide synthesis pathway on the other hand is an energy consuming, multistep pathway that uses glucose as the starting material and undergoes more than 20 steps of enzymatic reactions to become ATP or GTP (Figure 1). In order to generate one molecule of GTP from glucose, nine ATP-coupled reactions, a glycine, two folates, two glutamines, and an aspartate are required. Interestingly, the energy consuming de novo purine nucleotide synthesis pathway is often employed by rapidly proliferating cancer cells, while the energy efficient salvage pathway is predominantly used by quiescent, noncancerous cells. This may be due in part to the preferential increase in activity of the enzymes of the de novo purine nucleotide synthesis pathway in tumor cells including IMPDH $[6,7]$.

2.2. Various Tumors Increase IMPDH Expression. IMPDH is the rate-limiting enzyme of the de novo purine nucleotide synthesis pathway and a molecular determinant of the fate of IMP to GTP synthesis (Figure 1). The human genome encodes two different IMPDH genes [8]. IMPDH1 is believed to be ubiquitously expressed, whereas IMPDH2 is expressed in proliferative states where it has been linked to cell growth and malignant transformation $[9,10]$. IMPDH1 is highly 

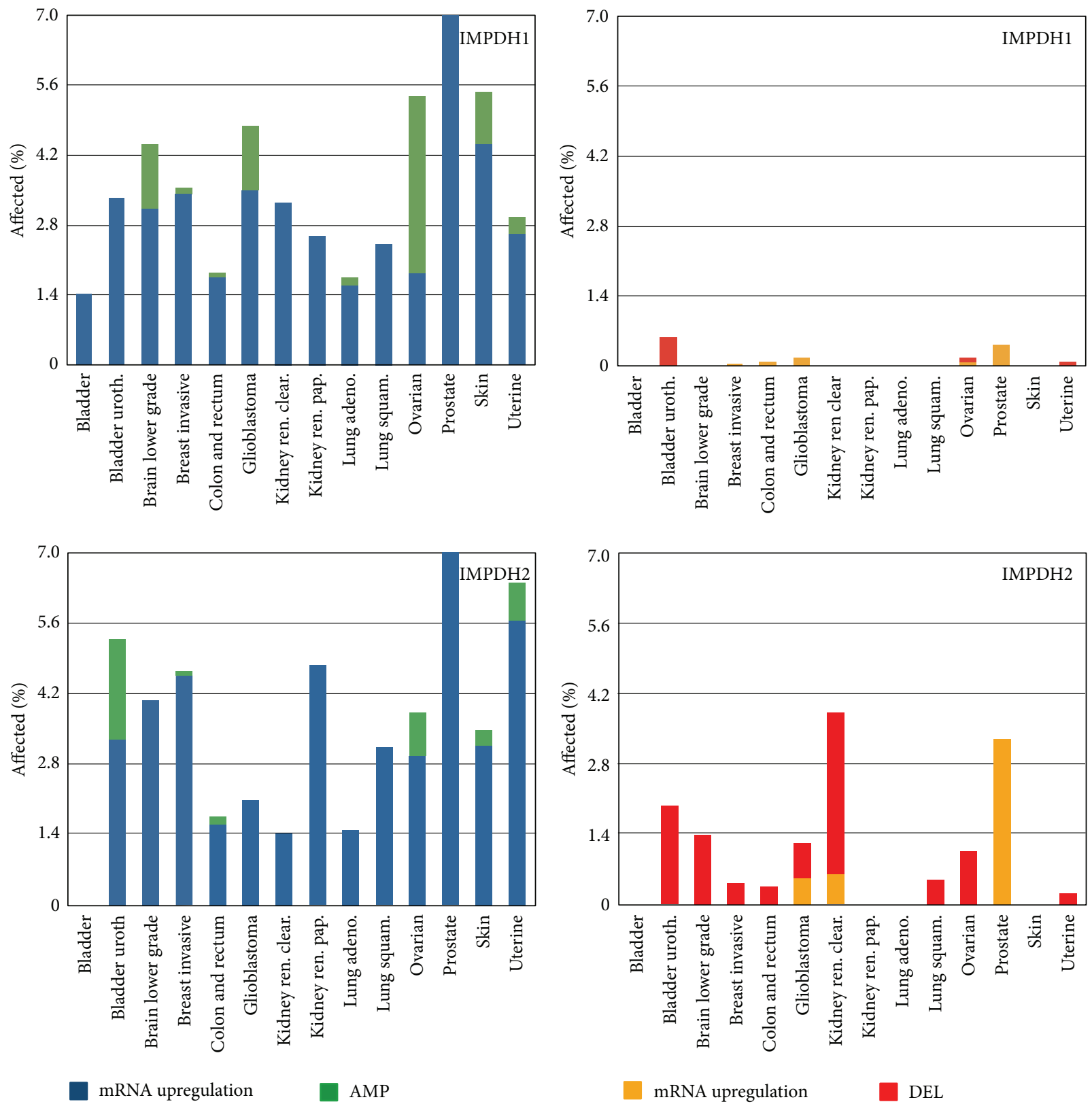

FIGURE 2: IMPDH gene alteration in cancer patients. AMP: amplification. DEL: deletion.

expressed in resting and activated peripheral blood lymphocytes as well as the retina [11, 12]. Mutations in unique isoforms of IMPDH1 expressed in retinal cells are associated with retina-specific photoreceptor degeneration suggesting that IMPDH1 is important for normal retinal development [12].

To gain insight into altered IMPDH expression in tumors, we analyzed the cBio database operated by Memorial Sloan Kettering Cancer Center (http://www.cbioportal.org/publicportal/cross_cancer.do) that provides gene expression profiles in various cancer patient tissues. Figure 2 demonstrates IMPDH1 and IMPDH2 gene amplification and mRNA upregulation in a number of tumors obtained from cancer patients including brain, ovarian, skin, bladder, renal cell, and uterine cancers. It should be noted that some tumors have reduced IMPDH expression, though it is a minor fraction. This suggests that some tumors may use IMPDHindependent nucleotide synthesis pathways. In vitro studies have corroborated the preferential upregulation of IMPDH in tumors obtained from patients in that many tumor cell lines also carry high levels of IMPDH expression (summarized in Table 1). These analyses show that IMPDH gene expression is elevated in a number of tumors including brain, sarcoma, and leukemia, when compared to normal tissue counterparts. Specifically, Fellenberg et al. showed that IMPDH2 gene expression is significantly elevated in osteosarcoma patients with poor response to chemotherapy and that this elevated expression is associated with poor risk disease evidenced 
TABLE 1: IMPDH gene overexpression in a number of malignancies.

\begin{tabular}{lrr}
\hline Malignancy/cell lines & IMPDH & References \\
\hline Osteosarcoma patients with poor response to chemotherapy & Elevated expression & Fellenberg et al., 2007 [13] \\
\hline Osteosarcoma cells (SaO2 ) & $\begin{array}{c}\text { Exogenous overexpression induced } \\
\text { chemoresistance }\end{array}$ & Fellenberg et al., 2010 [9] \\
\hline MTX-resistant erythroleukemia cells (K562) & Overexpressed & Peñuelas et al., 2005 [15] \\
\hline MTX-resistant human colon cancer cells (HT29) & Overexpressed & Peñuelas et al., 2005 [14] \\
\hline Leukemic cells (HL-60) & (i) Upregulated by induced proliferation & Nagai et al., 1992 [16] \\
\hline
\end{tabular}

MTX: methotrexate.

by decreased event-free survival [13]. Similarly, Peñuelas et al. showed that IMPDH2 is overexpressed in methotrexateresistant erythroleukemia cells, K562, and human colon cancer cells, HT29 $[14,15]$. IMPDH2 mRNA is also selectively overexpressed in leukemic cancer cells $[16,17]$. In HL-60 leukemic cells, IMPDH2 mRNA expression is upregulated by 2.8 -fold under conditions of increased proliferation and downregulated to less than $5 \%$ by induced differentiation [16]. These data demonstrate that both patient-derived tumor tissue and cultured cell lines result in elevated IMPDH expression levels.

This IMPDH mediated GTP biosynthesis pathway is preferentially used by rapidly proliferating cells over the salvage pathway to support increased RNA production and DNA replication needed by transformed cells [7]. It is not yet clear how IMPDH levels are upregulated in cancer cells. In experiments using yeast, the researchers have found that expression level of yeast IMPDH homologues mRNA and protein are increased upon depletion of cellular GTP by mycophenolate treatment and reduced by GTP replenishment [18, 19]. Clearly, this induction of IMPDH genes observed in yeast is a likely homeostatic mechanism to increase GTP production. While it remains elusive whether this regulation of human IMPDH genes is conserved throughout evolution, a potential model could be that cancer cells encounter GTP depletion due to the increase in anabolic processes, which leads to the induction of IMPDH genes.

However, the above model may not explain the observation that elevation of IMPDH is associated with elevated GTP levels in cancer cells [20, 21]. Furthermore, it has been postulated that IMPDH elevation in tumor cells may be due to upstream regulators including p210 bcr-abl protein [22]. Increased GTP levels in tumor cells can at least in part be due to elevated IMPDH levels [5].

\section{Is GTP Upregulation Important for Tumor Cell Growth?}

Alterations in IMPDH genes including amplification and deletion as well as varying mRNA expressions are seen in a wide variety of cancer patients' tissues and a number of cancer cell lines (Figure 2 and Table 1). In this section, we review studies that evaluate the role of increased GTP levels in tumor cells. Many of these studies use a pharmacological inhibitor of IMPDH to modulate cellular GTP levels.

3.1. MPA, the Active Form of MMF, Is a Potent, Highly Selective, Pharmacological Inhibitor of the De Novo GTP Synthesis Pathway. Mycophenolic acid (MPA) is the active form of MMF. MMF is hydrolyzed to its active form, MPA, in the gut wall, blood, and liver. MMF is the compound administered to patients and MPA is the compound more commonly used in in vitro and in vivo experiments. These names have been used distinctly within the paper depending on the type of studies that are described.

MPA is an inhibitor of IMPDH that inhibits conversion of IMP to XMP (Figure 1) and results in cellular GTP reduction. The bioavailability of MPA after oral administration of MMF is more than $90 \%$ [23]. MMF inhibits proliferation of both $\mathrm{T}$ and $\mathrm{B}$ lymphocytes and has been widely used in posttransplant medicine for prevention of acute and chronic allograft rejection since its introduction as an immunosuppressant in 1994. MMF is also used as an immunomodulator to treat an array of autoimmune conditions such as lupus nephritis, idiopathic thrombocytopenic purpura, and scleroderma [24]. Widespread use of MMF in the field of organ transplantation has demonstrated a favorable safety profile [25]. In addition to its effect on the immune system, the role of MMF as an antitumor drug has been investigated.

3.2. MPA Suppresses Tumor Cell Growth In Vitro and In Vivo. The very first reports of antiproliferative effects of MPA were published in the late 1960s [26]. Since then, the antitumor effect of MPA has been studied in cell lines obtained from a number of different malignancies. In these studies, MPA treatment suppresses cell proliferation of leukemia, lymphoma, pancreatic cancer, non-small-cell lung adenocarcinoma, and colon cancer cell lines as summarized in Table 2 [27-37].

In addition, murine models have been studied to test the potential tumor suppressive effect of MMF in vivo. Immunodeficient nude mice were subcutaneously injected with human cancer cell lines, A3.01 (T-lymphoblast), Molt4 (T-cell leukemia), CaPan-2 (pancreatic adenocarcinoma), CaLu-3 (non-small-cell lung adenocarcinoma), LS174T and T84 (colon adenocarcinoma), and Daudi (B-cell lymphoma). 


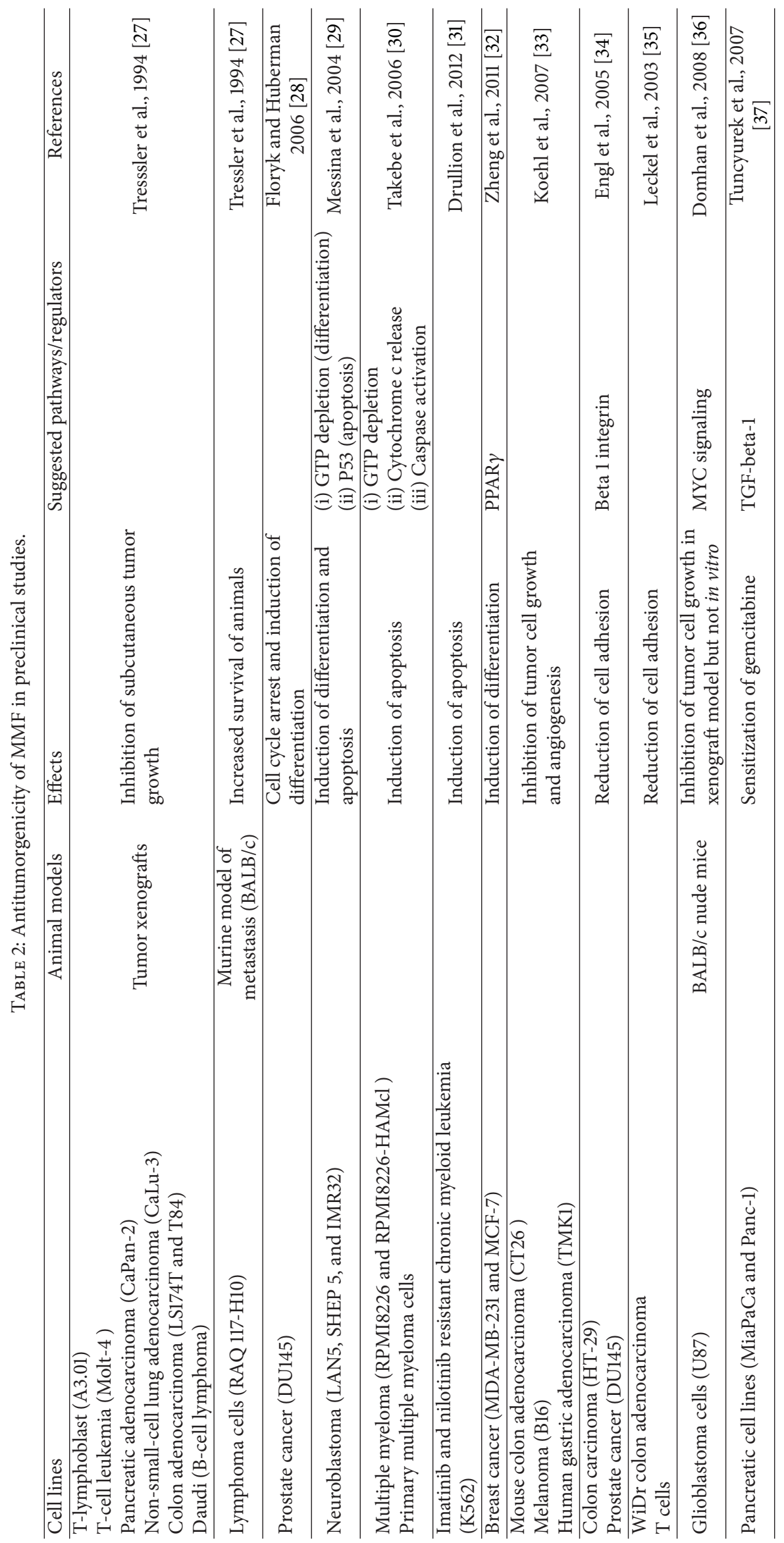


The mice were treated with oral MMF either 24 hours prior to tumor injection or after tumors became palpable. MMF significantly inhibited subcutaneous tumor growth in both groups regardless of time of administration. The injected MMF dose was 30,120 , or $240 \mathrm{mg} / \mathrm{kg} /$ day in two daily doses. There was a dose response curve in the majority of cell lines tested with the doses of 120 or $240 \mathrm{mg} / \mathrm{kg} /$ day showing statistically significant reduction in xenograft tumor size. Additionally, MMF treatment in a murine model of metastasis, (BALB/c) injected with RAW 117-H10 lymphoma cells resulted in increased survival compared to controls [27]. Here, only the $240 \mathrm{mg} / \mathrm{kg} /$ day dosing was active in prolonging survival. The above studies demonstrate that MMF may be a feasible and efficacious anticancer therapy.

\subsection{MPA Suppresses Tumor Cell Growth via Diverse Mech-} anisms. As shown in Table 2, MPA treatment leads to suppression of tumor cell growth in many cell lines. Interestingly, the mechanism of growth suppression by MPA shows significant diversity: (1) replication arrest (arrest at S-phase of cell cycle) in DU145 prostate cancer cell lines [28], (2) induction of tumor cell differentiation in neuroblastoma cell lines (LAN5, SHEP5, and IMR32) [29], (3) p53-mediated G1 cell cycle arrest and apoptosis of neuroblastoma cell lines (LAN5, SHEP5, and IMR32) [29], (4) attenuation of cell cycle progression and apoptosis following concomitant cytochrome c release and caspase activation of multiple myeloma cell lines and primary myeloma cells [30], and (5) apoptosis or cellular senescence of chronic myeloid leukemia cells [31]. Currently, the mechanism by which MPA treatment suppresses cell growth via such diverse pathways is unclear. It is likely that such differential MPA responses are attributed to the differential genetic mutations and/or cellular metabolism in each cancer type. It is important to clarify the molecular mechanism by which MPA exerts its cell growth suppressive effects to further test this drug in clinical trials.

\section{Does Targeting GTP Synthesis Pathway Result in Antitumorigenesis?}

Next, we review the literature investigating other antitumorigenic effects of MPA, including tumor cell differentiation, angiogenesis, and cell adhesion.

4.1. MPA Induces Tumor Cell Differentiation. Messina and colleagues showed that MPA induces differentiation of human neuroblastoma cell lines (LAN5, SHEP 5, and IMR32) by causing partial chronic guanine nucleotide depletion. They also demonstrated that MPA leads to cell cycle arrest and apoptosis through a p53-mediated pathway [29]. Similar studies were performed in breast and prostate cancer cell lines. MPA treatment of breast cancer cell lines (MDA-MB231 and MCF-7) resulted in differentiation of adipose tissue as evidenced by arrest of breast cancer cells at the G1/G0 stage of the cell cycle, adipocyte-like morphological conversion, and lack of colony formation in treated breast cancer cells. Additionally, MPA resulted in upregulation of three adipocyte differentiation markers, PPAR $\gamma$, adipsin $\mathrm{D}$, and $\mathrm{aP} 2$. This differentiation was thought to be partly due to PPAR $\gamma$ activation since the RNAi knockdown of PPAR $\gamma$ partially reversed the differentiated state of MDA-MB-231 cells after MPA treatment [32]. In the DU145 prostate cancer cell line, MPA induced expression of a number of differentiation markers including CD55, clusterin, granulophysin, glucoserelated protein 78, vasoactive intestinal polypeptide, and prostate-specific transglutaminase [28]. Poorly differentiated status of tumor is associated with aggressive phenotypes. Thus, these data suggest that treatment with MPA may be an active anticancer therapy by inducing differentiation in tumor cells resulting in cell cycle arrest.

4.2. MMF Inhibits Angiogenesis. Neovascularization, also known as angiogenesis, is critical for tumors to maintain a high level of nutrient uptake and proliferation. MMF was discovered as a potent inhibitor of angiogenesis when Chong and colleagues screened a library of 1850 FDA approved drugs. They screened for inhibition of human umbilical vein endothelial cells (HUVEC) using $\mathrm{H} 3$ thymidine incorporation. MPA was discovered to be a potent inhibitor of HUVEC proliferation with an IC50 of about $100 \mathrm{nM}$. IMPDH1 was the predominantly expressed isoform in these endothelial cells and knockdown of its expression by RNAi was sufficient to cause endothelial cell cycle arrest in G1 [38].

In addition, MMF was shown to inhibit neovascularization in an aortic ring assay [33]. In this study, slices of rat aorta were cultured on matrigel-coated 24-well plates where sprouts from the aortic rings are normally visible after a few days. MMF at concentrations from 1 to $100 \mathrm{uM}$ almost completely inhibited sprouting from the aortic rings. The same group showed that MMF causes a dose-dependent cell growth reduction in mouse CT26 colon adenocarcinoma, B16 melanoma, and human TMK1 gastric adenocarcinoma cells in vitro [33].

4.3. MMF Modulates Tumor Cell Adhesion. MMF prevents allograft rejection via blocking the proliferation of $\mathrm{T}$ and $\mathrm{B}$ lymphocytes. Another mechanism of preventing allograft rejection by MMF is via suppressing the expression of adhesion molecules and decreasing the recruitment of immune cells into sites of graft rejection [39]. Given that regulation of adhesion molecules is pivotal in the ability of tumor cells to metastasize, it is conceivable that MMF could affect invasion of tumor cells. It has been shown that MPA modulates adhesion properties of the colon carcinoma cell line, HT-29, and prostate tumor cell line, DU-145 [34]. Reduction in adhesion of HT-29 to endothelial cells by MPA is accompanied by downregulation of alpha subunits of beta 1 integrin-an adhesive protein whose varying expressions are associated with tumor cell growth and metastasis. On the other hand, reduction in adhesion of DU-145 cells is accompanied by upregulation of beta 1 subtypes. Upregulation of integrin subtypes in the face of decreased adhesion of cells in DU-145 cells could possibly be secondary to redifferentiation towards low-invasive phenotypes [34].

In another study, modulation of adhesion molecules by MMF was thought to increase the risk of tumor metastasis [40]. Blaheta and colleagues showed that MMF decreases 
neural cell adhesion molecule (NCAM) receptors in neuroblastoma cells that self-aggregate by means of the homophilicbinding element. Decreased number of NCAM receptors is associated with increased migratory potential of neuroblastoma cells. It is inquiring that modulation of different cell adhesion molecules results in varying outcomes, redifferentiation of cells towards less invasive phenotypes versus promotion of migratory potential of cells. This likely depends on the specific cell adhesion molecules that are expressed on individual types of cancer cells.

Other groups have shown that MMF suppresses expression of additional adhesion molecules such as lymphocytic alpha4betal integrin [39, 41]. Additionally, MMF was shown to decrease T-cells and WiDr colon adenocarcinoma cell line binding to adhesion endothelium by $80 \%$ [35]. Here, MMF potently blocked both T-cells binding to ICAM-1, VCAM-1, and P-selectin and prevented WiDr cell attachment to E-selectin [35]. These results suggest that MPA could modulate cell adhesion via multiple molecular pathways. In addition, Dun and colleagues demonstrated that MPA has antimigratory and anti-invasive properties in gastric cancer cells via downregulation of large number of promigratory proteins such as AKT and MMP1 [42].

Consistent with the notion that MPA affects tumor microenvironment, Domhan and colleagues showed that U87 glioblastoma cells are resistant to MPA treatment in vitro, but their growth is markedly inhibited in vivo in $\mathrm{BALB} / \mathrm{c}$ nude mice [36]. Genome-wide transcriptional analysis in U87, endothelial and fibroblast cells treated with MPA revealed a role for MYC signaling in endothelial cells.

In addition to the above-noted antitumor effects, MMF is effective as a chemotherapeutic agent in pancreatic cancer in vitro. In $\mathrm{MiaPaCa}$ and Panc-1 pancreatic cell lines, concomitant administration of MMF increased the EC50 response of gemcitabine treatment that is widely used in patients with pancreatic adenocarcinoma. In addition, treatment of Panc1 cells with MMF inhibited TGF-beta-1 induced collagen I expression in a dose-dependent manner [37]. It has been shown that collagen I promotes metastasis in pancreatic cancer cells by activating c-Jun and upregulation of $\mathrm{N}$ cadherin expression [43]. These studies concluded that MMF inhibition of collagen I overexpression may be responsible for the reduction seen in pancreatic cell line invasiveness.

\section{Clinical Studies of MMF as an Anticancer Drug}

Finding of MMF as an antitumor agent in vitro and in vivo has lead to its use in a number of clinical trials. MMF is already an FDA-approved drug, and its tolerability profile is superior to many other commonly used chemotherapeutic agents.

The first clinical trial involving MMF as an anticancer therapeutic was performed in multiple myeloma patients. In this phase I dose escalation study, MMF was well tolerated up to $5 \mathrm{~g} /$ day daily doses in 11 patients with relapsed and refractory disease [44]. Interestingly, the intracellular dGTP level in peripheral blood mononuclear cells was decreased in five patients with partial response or stable disease in comparison with the six patients that had progression of disease. This finding was at least in part consistent with the known mechanism of action of MMF: GTP depletion by MMF leads to alteration of intracellular guanine pool resulting in nucleotide imbalance and hampered DNA synthesis.

Rodríguez-Pascual and colleagues performed a high throughput screening for anticancer activity of FDA approved drugs and identified MPA as an active antiangiogenic agent. Here, MPA resulted in growth inhibition and reduced expression of vascular endothelial growth factor (VEGF) that was reversed by guanosine supplementation in the media in vitro [45]. Additionally, MPA was shown to have antitumor effects in a panel of six human derived pancreatic cancer xenografts in immunodeficient mice. They therefore tested MPA in a pilot clinical study in which MMF was administered to twelve patients with resectable pancreatic cancer prior to resection. However, assessment of VEGF levels and an apoptosis marker in resected specimens in resected specimens compared to six untreated patients showed no significant difference in expression [45]. Survival analysis was not reported. In this study, FDA-approved immunosuppressive doses were used, 1 or 2 gram per day of MMF divided into two daily doses. Higher doses might have shown more promising results, but the purpose of this study was to use dosages that would not require additional safety testing. Further studies will be required to determine efficacy of MMF in pancreatic adenocarcinoma.

\section{Tolerability and Bioavailability of Mycophenolate}

Two mycophenolate compounds are commercially available, mycophenolate mofetil (MMF) and enteric-coated mycophenolate sodium (EC-MFS). Mycophenolate compounds are the prodrug of MPA. MPA, the active form of mycophenolate, has a complex pharmacokinetic profile; it undergoes glucuronidation, enterohepatic recirculation, and proteindependent clearance. The pharmacokinetics of MPA and MMF are not linear in transplant patients [46]. MMF, the ester prodrug of MPA, was developed to improve MPA bioavailability [47]. The increase in bioavailability was linked to increased gastrointestinal (GI) intolerance. However, more recent studies point towards improved GI complications (particularly diarrhea) with the use of enteric-coated mycophenolate sodium in posttransplant patients [48-50]. GI intolerance as well as other serious complications of MPA such as leukopenia and anemia seems to be dose dependent [51].

Bioavailability of MPA after MMF administration is about $90 \%$ versus $74 \%$ for EC-MFS [23, 51, 52]. MPA is highly protein bound (97-99\%) and therefore its total and free concentrations are subject to interpretation in patients with hypoalbuminaemia (i.e., severe liver or renal disease as well as cancer patients with severe malnutrition or impaired liver synthetic function). Given that MPA undergoes glucuronidation, its effective dose may be reduced in the setting of induced expression of glucuronosyltransferases in the setting of high dose corticosteroids. Cyclosporine reduces enterohepatic recirculation of MPA and therefore lowers its effective dose. Most pharmacokinetic studies of MPA 
have been obtained in posttransplant patients with varying albumin concentrations and immunosuppressant cotherapy [53-55]. Lessons learned from transplant literature call for careful monitoring of MPA total and free levels as well as potentially monitoring other biomarkers such as IMPDH levels when and if MMF is widely used in cancer patients.

\section{Safety of MMF in Post-Transplant Patients}

The introduction of immunosuppressive agents for treatment of acute rejection in post-transplant patients has led to prolonged survival and the advent of immunosuppressantinduced malignancies [56]. MMF was amongst the immunosuppressive agents initially thought to be the culprit of posttransplant malignancy. However, transplant patients are very rarely treated with MMF monotherapy. Other immunosuppressive drugs, most commonly calcineurin inhibitors, are usually part of the treatment regimen and have been shown to increase the rate of nonmelanocytic skin cancer and lymphoma in posttransplant patients [57].

The relative risk of post-transplant malignancy seems to be lower with the use of MMF than other immunosuppressive drugs such as calcineurin inhibitors and azathioprine [58, 59]. However, patients were on multidrug regimens rather than MMF monotherapy in these studies making it difficult to decipher the actual contribution of each agent. Robson et al. designed a prospective observational cohort study comparing 6751 patients from two different renal transplant registries receiving $\mathrm{MMF}$ with an equal number of matched controls receiving non-MMF immunosuppressants. This study did not show an association between the use of MMF and an increased risk of lymphoma with follow-up of 3 years. Interestingly, in the MMF group, there was a trend toward a lower risk of any malignancy [58]. Although prospective, this study was limited in that patients were only followed for 3 years after transplant for development of malignancy. This study was corroborated in a recent retrospective single-center cohort of 929 adult renal transplant recipients [60]. The latter study showed that MMF used for prevention of acute rejection after kidney transplant was not associated with an increased risk of solid tumors or posttransplant lymphoproliferative disorders. A similar study was performed in liver transplant patients. Using Scientific Registry of Transplant Recipients between 1995 and 2004, triple immunosuppressive regimen involving MMF, tacrolimus, and corticosteroids $(n=9180)$ was compared with dual therapy, tacrolimus and corticosteroids $(n=10,099)$. There was no significant difference in death due to malignancy between patients 4 years after transplant and the treatment groups. The incidence of posttransplant malignancy was also not significantly different. Therefore, addition of MMF to the dual therapy (tacrolimus plus corticosteroids) was not associated with an increased risk of malignancy in liver transplant recipients at 4 years [61]. Overall, as noted above, the analysis of data involving large groups of patients in posttransplant registries has failed to show an increased risk of malignancy secondary to MMF.

It is worth noting that immunosuppressive reagents may increase the risk of oncogenic viral infections. For example, in the nontransplant literature where MMF is used as monotherapy, there have been few reports of Epstein-Barr virus associated central nervous system lymphoma that were thought to be secondary to prolonged use of MMF [62-65]. However, it is reassuring that studies involving large posttransplant registries did not indicate an association between increased malignancy rate and the use of MMF directly.

\section{Other IMPDH Inhibitors}

In addition to MMF, other IMPDH inhibitors were tested in preclinical and phase I/II clinical trials as summarized in Table 3 [66-73]. A newer agent AVN-944, also known as VX-944, was developed due to concerns about MMF potency and gastrointestinal tolerability. This agent was well tolerated in normal volunteers in a phase I study, but it was not further developed due to financial issues [71, 73]. Most other IMPDH inhibitors such as tiazofurin were found to have robust antitumorigenic effects in preclinical and phase I/II clinical trials; however, further studies were limited due to their side effects [68] (Table 3).

\section{Discussion}

The de novo purine nucleotide synthesis pathway is upregulated in many tumors and is essential for their growth. Modulation of the GTP de novo synthesis pathway by inhibition of IMPDH, the rate-limiting enzyme for GTP biosynthesis, seems to be a promising approach in our endeavors to inhibit cancer cell growth. MMF, an FDA-approved immunosuppressant commonly used for prevention of acute transplant rejection and treatment of autoimmune conditions, has been studied as an antitumor drug in several tumor cell lines. Preclinical studies have suggested multiple antitumorigenic effect of MMF including (1) inhibition of cell proliferation, (2) inhibition of angiogenesis, (3) inhibition of cell adhesion, and (4) induction of cellular differentiation. These findings are likely due to the various roles of G-proteins in different mammalian cells. It is important to decipher the precise molecular mechanism of MMF to further guide its use in specific tumor types.

The safety and tolerability of MMF have been confirmed in multiple phase I clinical trials. We believe that it is opportune time to further investigate MMF as an antitumor drug using mouse orthotopic tumor models and genetically engineered models that more precisely reflect the human tumor environment. Multiple preclinical trials have shown that MMF antitumor effects are reversed by addition of guanine to the cells. This suggests the possibility that MMF antitumor effects are related to its ability to inhibit the de novo purine synthesis pathway presumably via inhibition of IMPDH. A potential caveat of the use of MMF as an antitumor drug is that GTP can be produced via the de novo pathway as well as the compensatory salvage pathway. Tumor cell lines have the potential to develop resistance to MMF by upregulating the salvage pathway after prolonged treatment. Therefore, it would be beneficial to measure the cellular and blood guanine nucleotide concentrations in future studies that investigate the antitumor role of MMF in vivo and MPA in vitro and to potentially combine MMF with other treatment approaches. 
TABLE 3: Antitumorigenicity of other IMPDH inhibitors.

\begin{tabular}{|c|c|c|c|}
\hline IMPDH inhibitors & Study design: results & Suggested pathways & References \\
\hline \multirow{3}{*}{ Tiazofurin } & $\begin{array}{l}\text { Preclinical: } \\
\text { activation of erythroid differentiation program of } \\
\text { human leukemia K-562 cells }\end{array}$ & $\begin{array}{l}\text { Downregulation of } \\
\text { c-ras gene }\end{array}$ & $\begin{array}{l}\text { Gebeyehu et al., } \\
1985 \text { [66] } \\
\text { Olah et al., } 1988 \\
{[67]}\end{array}$ \\
\hline & $\begin{array}{l}\text { Phase I: } \\
\text { - } 16 \text { patients with nonlymphocytic leukemia or } \\
\text { myeloid blast crisis of chronic granulocytic } \\
\text { leukemia. } \\
\quad \text { } 8 \text { patients showed improvement or complete } \\
\text { remission. }\end{array}$ & & $\begin{array}{c}\text { Tricot et al., } \\
1989[68]\end{array}$ \\
\hline & $\begin{array}{l}\text { Phase II: } \\
\text { - } 6 \text { patients with bcr-abl positive AML or CML in } \\
\text { blast crisis } \\
\quad \text { Transient hematological response }\end{array}$ & & $\begin{array}{l}\text { Malek et al., } \\
2004[69]\end{array}$ \\
\hline Benzamide riboside & $\begin{array}{l}\text { Preclinical: } \\
\text { induction of apoptosis in human lung cancer } \\
\text { H520 cells }\end{array}$ & $\begin{array}{l}\text { Increased caspase } 3 \\
\text { activity and PAPR } \\
\text { cleavage }\end{array}$ & $\begin{array}{l}\text { Khanna et al., } \\
2004 \text { [70] }\end{array}$ \\
\hline \multirow[t]{2}{*}{ AVN-944 } & $\begin{array}{l}\text { Preclinical: } \\
\text { inhibition of cell proliferation in prostate cancer } \\
\text { cell lines }\end{array}$ & Cell cycle arrest & $\begin{array}{l}\text { Floryk and } \\
\text { Thompson, } \\
2008[71]\end{array}$ \\
\hline & $\begin{array}{l}\text { Phase I: } \\
\text { well tolerated in } 25 \text { healthy males }\end{array}$ & & $\begin{array}{l}\text { Hamilton et al., } \\
2009[72]\end{array}$ \\
\hline VX-944 & $\begin{array}{l}\text { Preclinical: } \\
\text { induction of apoptosis in multiple myeloma cell } \\
\text { lines }\end{array}$ & $\begin{array}{l}\text { Caspase-independent } \\
\text { pathway }\end{array}$ & $\begin{array}{l}\text { Ishitsuka et al., } \\
2005[73]\end{array}$ \\
\hline
\end{tabular}

\section{Conclusion}

Mycophenolate mofetil is an FDA-approved immunosuppressant with antitumor effects. Its use as antitumor drug should be revisited in light of its potential for treatment of malignancies that overexpress IMPDH and its favorable safety profile in comparison with other chemotherapeutic agents. Careful research on the role of GTP regulation in tumor cells can aid further clinical investigations on the role of MMF as an antitumor drug.

\section{Conflict of Interests}

The authors confirm that there is no conflict of interests regarding this publication.

\section{Acknowledgment}

This work was partially supported by Mayfield Education Research Foundation Cincinnati (Nazanin Majd and Atsuo T. Sasaki). KS was supported, in part, by the American Association of Neurological Surgeons. HY was supported, in part, by the Uehara Memorial Foundation. Funding was also provided by grants from the UC-Brain Tumor Center (Atsuo T. Sasaki), and the Marlene Harris-Ride Cincinnati foundation (Atsuo T. Sasaki and Jessica Guarnaschelli).

\section{References}

[1] P. Liu, H. Cheng, T. M. Roberts, and J. J. Zhao, "Targeting the phosphoinositide 3-kinase pathway in cancer," Nature Reviews Drug Discovery, vol. 8, no. 8, pp. 627-644, 2009.

[2] R. A. Cairns, I. S. Harris, and T. W. Mak, "Regulation of cancer cell metabolism," Nature Reviews Cancer, vol. 11, no. 2, pp. 8595, 2011.

[3] K. H. Khan, T. A. Yap, L. Yan, and D. Cunningham, "Targeting the PI3K-AKT-mTOR signaling network in cancer," Chinese Journal of Cancer, vol. 32, no. 5, pp. 253-265, 2013.

[4] J. S. Carew, K. R. Kelly, and S. T. Nawrocki, "Mechanisms of mTOR inhibitor resistance in cancer therapy," Targeted Oncology, vol. 6, no. 1, pp. 17-27, 2011.

[5] T. W. Traut, "Physiological concentrations of purines and pyrimidines," Molecular and Cellular Biochemistry, vol. 140, no. 1, pp. 1-22, 1994.

[6] Y. Natsumeda, N. Prajda, J. P. Donohue, J. L. Glover, and G. Weber, "Enzymic capacities of purine de novo and salvage pathways for nucleotide synthesis in normal and neoplastic tissues," Cancer Research, vol. 44, no. 6, pp. 2475-2479, 1984.

[7] X. Tong, F. Zhao, and C. B. Thompson, "The molecular determinants of de novo nucleotide biosynthesis in cancer cells," Current Opinion in Genetics \& Development, vol. 19, no. 1, pp. 32-37, 2009. 
[8] Y. Natsumeda, S. Ohno, H. Kawasaki, Y. Konno, G. Weber, and K. Suzuki, "Two distinct cDNAs for human IMP dehydrogenase," The Journal of Biological Chemistry, vol. 265, no. 9, pp. 5292-5295, 1990 .

[9] J. Fellenberg, P. Kunz, H. Sähr, and D. Depeweg, "Overexpression of inosine 5'-monophosphate dehydrogenase type II mediates chemoresistance to human osteosarcoma cells," PLoS ONE, vol. 5, no. 8, Article ID e12179, 2010.

[10] C. Brouwer, D. G. M. Vermunt-de Koning, R. C. Trueworthy et al., "Monitoring of inosine monophosphate dehydrogenase activity in mononuclear cells of children with acute lymphoblastic leukemia: enzymological and clinical aspects," Pediatric Blood and Cancer, vol. 46, no. 4, pp. 434-438, 2006.

[11] J. Jain, S. J. Almquist, P. J. Ford et al., "Regulation of inosine monophosphate dehydrogenase type I and type II isoforms in human lymphocytes," Biochemical Pharmacology, vol. 67, no. 4, pp. 767-776, 2004.

[12] S. J. Bowne, Q. Liu, L. S. Sullivan et al., "Why do mutations in the ubiquitously expressed housekeeping gene IMPDH1 cause retina-specific photoreceptor degeneration?" Investigative Ophthalmology and Visual Science, vol. 47, no. 9, pp. 3754-3765, 2006.

[13] J. Fellenberg, L. Bernd, G. Delling, D. Witte, and A. ZahltenHinguranage, "Prognostic significance of drug-regulated genes in high-grade osteosarcoma," Modern Pathology, vol. 20, no. 10, pp. 1085-1094, 2007.

[14] S. Peñuelas, V. Noé, and C. J. Ciudad, "Modulation of IMPDH2, survivin, topoisomerase I and vimentin increases sensitivity to methotrexate in HT29 human colon cancer cells," FEBS Journal, vol. 272, no. 3, pp. 696-710, 2005.

[15] S. Peñuelas, V. Noé, R. Morales, and C. J. Ciudad, "Sensitization of human erythroleukemia K562 cells resistant to methotrexate by inhibiting IMPDH," Medical Science Monitor, vol. 11, no. 1, pp. BR6-BR12, 2005.

[16] M. Nagai, Y. Natsumeda, and G. Weber, "Proliferation-linked regulation of type II IMP dehydrogenase gene in human normal lymphocytes and HL-60 leukemic cells," Cancer Research, vol. 52, no. 2, pp. 258-261, 1992.

[17] M. Nagai, Y. Natsumeda, Y. Konno, R. Hoffman, S. Irino, and G. Weber, "Selective up-regulation of type II inosine $5^{\prime}$ monophosphate dehydrogenase messenger RNA expression in human leukemias," Cancer Research, vol. 51, no. 15, pp. 38863890, 1991.

[18] M. Escobar-Henriques, A. Balguerie, C. Monribot, H. Boucherie, and B. Daignan-Fornier, "Proteome analysis and morphological studies reveal multiple effects of the immunosuppressive drug mycophenolic acid specifically resulting from guanylic nucleotide depletion," The Journal of Biological Chemistry, vol. 276, no. 49, pp. 46237-46242, 2001.

[19] J. N. Kuehner and D. A. Brow, "Regulation of a eukaryotic gene by GTP-dependent start site selection and transcription attenuation," Molecular Cell, vol. 31, no. 2, pp. 201-211, 2008.

[20] K. Pillwein, P. Chiba, A. Knoflach et al., "Purine metabolism of human glioblastoma in vivo," Cancer Research, vol. 50, no. 5, pp. 1576-1579, 1990.

[21] H. N. Jayaram, D. A. Cooney, M. Grusch, and G. Krupitza, "Consequences of IMP dehydrogenase inhibition, and its relationship to cancer and apoptosis," Current Medicinal Chemistry, vol. 6, no. 7, pp. 561-574, 1999.
[22] K. Gharehbaghi, G. S. Burgess, F. R. Collart et al., "P210 BcrAbl confers overexpression of inosine monophosphate dehydrogenase: an intrinsic pathway to drug resistance mediated by oncogene," Leukemia, vol. 8, no. 8, pp. 1257-1263, 1994.

[23] R. Bullingham, S. Monroe, A. Nicholls, and M. Hale, "Pharmacokinetics and bioavailability of mycophenolate mofetil in healthy subjects after single-dose oral and intravenous administration," Journal of Clinical Pharmacology, vol. 36, no. 4, pp. 315-324, 1996.

[24] R. A. Moore and S. Derry, "Systematic review and meta-analysis of randomised trials and cohort studies of mycophenolate mofetil in lupus nephritis," Arthritis Research and Therapy, vol. 8, article R182, 2006.

[25] J. Zwerner and D. Fiorentino, "Mycophenolate mofetil," Dermatologic Therapy, vol. 20, no. 4, pp. 229-238, 2007.

[26] S. Suzuki, T. Kimura, K. Ando, M. Sawada, and G. Tamura, "Antitumor activity of mycophenolic acid," Journal of Antibiotics, vol. 22, no. 7, pp. 297-302, 1969.

[27] R. J. Tressler, L. J. Garvin, and D. L. Slate, "Anti-tumour activity of mycophenolate mofetil against human and mouse tumors in vivo," International Journal of Cancer, vol. 57, no. 4, pp. 568-573, 1994.

[28] D. Floryk and E. Huberman, "Mycophenolic acid-induced replication arrest, differentiation markers and cell death of androgen-independent prostate cancer cells DU145," Cancer Letters, vol. 231, no. 1, pp. 20-29, 2006.

[29] E. Messina, L. Barile, F. Lupi, and A. Giacomello, "Potential role of mycophenolate mofetil in the management of neuroblastoma patients," Nucleosides, Nucleotides and Nucleic Acids, vol. 23, no. 8-9, pp. 1545-1549, 2004.

[30] N. Takebe, X. Cheng, T. E. Fandy et al., "IMP dehydrogenase inhibitor mycophenolate mofetil induces caspase-dependent apoptosis and cell cycle inhibition in multiple myeloma cells," Molecular Cancer Therapeutics, vol. 5, no. 2, pp. 457-466, 2006.

[31] C. Drullion, V. Lagarde, R. Gioia et al., "Mycophenolic Acid overcomes imatinib and nilotinib resistance of chronic myeloid leukemia cells by apoptosis or a senescent-like cell cycle arrest," Leukemia Research and Treatment, vol. 2012, Article ID 861301, 9 pages, 2012.

[32] Z.-H. Zheng, Y. Yang, X.-H. Lu et al., "Mycophenolic acid induces adipocyte-like differentiation and reversal of malignancy of breast cancer cells partly through PPAR $\gamma$," European Journal of Pharmacology, vol. 658, no. 1, pp. 1-8, 2011.

[33] G. E. Koehl, F. Wagner, O. Stoeltzing et al., "Mycophenolate mofetil inhibits tumor growth and angiogenesis in vitro but has variable antitumor effects in vivo, possibly related to bioavailability," Transplantation, vol. 83, no. 5, pp. 607-614, 2007.

[34] T. Engl, J. Makarević, B. Relja et al., "Mycophenolate mofetil modulates adhesion receptors of the betal integrin family on tumor cells: impact on tumor recurrence and malignancy," BMC Cancer, vol. 5, article 4, 2005.

[35] K. Leckel, W.-D. Beecken, D. Jonas et al., "The immunosuppressive drug mycophenolate mofetil impairs the adhesion capacity of gastrointestinal tumour cells," Clinical and Experimental Immunology, vol. 134, no. 2, pp. 238-245, 2003.

[36] S. Domhan, S. Muschal, C. Schwager et al., "Molecular mechanisms of the antiangiogenic and antitumor effects of mycophenolic acid," Molecular Cancer Therapeutics, vol. 7, no. 6, pp. 1656-1668, 2008.

[37] P. Tuncyurek, J. M. Mayer, F. Klug et al., "Everolimus and mycophenolate mofetil sensitize human pancreatic cancer cells to 
gemcitabine in vitro: a novel adjunct to standard chemotherapy?" European Surgical Research, vol. 39, no. 6, pp. 380-387, 2007.

[38] C. R. Chong, D. Z. Qian, F. Pan et al., "Identification of type 1 inosine monophosphate dehydrogenase as an antiangiogenic drug target," Journal of Medicinal Chemistry, vol. 49, no. 9, pp. 2677-2680, 2006.

[39] A. C. Allison and E. M. Eugui, "Mycophenolate mofetil and its mechanisms of action," Immunopharmacology, vol. 47, no. 2-3, pp. 85-118, 2000.

[40] R. A. Blaheta, H. Bogossian, W.-D. Beecken et al., "Mycophenolate mofetil increases adhesion capacity of tumor cells in vitro," Transplantation, vol. 76, no. 12, pp. 1735-1741, 2003.

[41] U. Heemann, H. Azuma, P. Hamar, C. Schmid, N. Tilney, and T. Philipp, "Mycophenolate mofetil inhibits lymphocyte binding and the upregulation of adhesion molecules in acute rejection of rat kidney allografts," Transplant Immunology, vol. 4, no. 1, pp. 64-67, 1996.

[42] B. Dun, A. Sharma, Y. Teng et al., "Mycophenolic acid inhibits migration and invasion of gastric cancer cells via multiple molecular pathways," PLoS ONE, vol. 8, Article ID e81702, 2013.

[43] Y. Shintani, M. A. Hollingsworth, M. J. Wheelock, and K. R. Johnson, "Collagen I promotes metastasis in pancreatic cancer by activating c-Jun $\mathrm{NH} 2$-terminal kinase 1 and up-regulating $\mathrm{N}$ cadherin expression," Cancer Research, vol. 66, no. 24, pp. 1174511753, 2006.

[44] N. Takebe, X. Cheng, S. Wu et al., "Phase I clinical trial of the inosine monophosphate dehydrogenase inhibitor mycophenolate mofetil (Cellcept) in advanced multiple myeloma patients," Clinical Cancer Research, vol. 10, no. 24, pp. 8301-8308, 2004.

[45] J. Rodríguez-Pascual, P. Sha, E. García-García et al., "A preclinical and clinical study of mycophenolate mofetil in pancreatic cancer," Investigational New Drugs, vol. 31, no. 1, pp. 14-19, 2013.

[46] B. C. M. de Winter, R. A. A. Mathot, F. Sombogaard, A. G. Vulto, and T. van Gelder, "Nonlinear relationship between mycophenolate mofetil dose and mycophenolic acid exposure: implications for therapeutic drug monitoring," Clinical Journal of the American Society of Nephrology, vol. 6, no. 3, pp. 656-663, 2011.

[47] W. A. Lee, L. Gu, A. R. Miksztal, N. Chu, K. Leung, and P. H. Nelson, "Bioavailability improvement of mycophenolic acid through amino ester derivatization," Pharmaceutical Research, vol. 7, no. 2, pp. 161-166, 1990.

[48] P. Reinke, K. Budde, C. Hugo et al., "Reduction of gastrointestinal complications in renal graft recipients after conversion from mycophenolate mofetil to enteric-coated mycophenolate sodium," Transplantation Proceedings, vol. 43, no. 5, pp. 16411646, 2011.

[49] F. Ortega, A. Sánchez-Fructuoso, J. M. Cruzado et al., “Gastrointestinal quality of life improvement of renal transplant recipients converted from mycophenolate mofetil to enteric-coated mycophenolate sodium drugs or agents: mycophenolate mofetil and enteric-coated mycophenolate sodium," Transplantation, vol. 92, no. 4, pp. 426-432, 2011.

[50] D. K. Granger, "Enteric-coated mycophenolate sodium: results of two pivotal global multicenter trials," Transplantation Proceedings, vol. 33, no. 7-8, pp. 3241-3244, 2001.

[51] C. E. Staatz and S. E. Tett, "Clinical pharmacokinetics and pharmacodynamics of mycophenolate in solid organ transplant recipients," Clinical Pharmacokinetics, vol. 46, no. 1, pp. 13-58, 2007.
[52] M. D. Pescovitz, D. Conti, J. Dunn et al., "Intravenous mycophenolate mofetil: safety, tolerability, and pharmacokinetics," Clinical Transplantation, vol. 14, no. 3, pp. 179-188, 2000.

[53] B. A. Atcheson, P. J. Taylor, C. M. J. Kirkpatrick et al., "Free mycophenolic acid should be monitored in renal transplant recipients with hypoalbuminemia," Therapeutic Drug Monitoring, vol. 26, no. 3, pp. 284-286, 2004.

[54] B. Kaplan, H. U. Meier-Kriesche, G. Friedman et al., "The effect of renal insufficiency on mycophenolic acid protein binding," Journal of Clinical Pharmacology, vol. 39, no. 7, pp. 715-720, 1999.

[55] H.-U. Meier-Kriesche, L. M. Shaw, M. Korecka, and B. Kaplan, "Pharmacokinetics of mycophenolic acid in renal insufficiency," Therapeutic Drug Monitoring, vol. 22, no. 1, pp. 27-30, 2000.

[56] J. F. Buell, T. G. Gross, and E. S. Woodle, "Malignancy after transplantation," Transplantation, vol. 80, no. 2, pp. S254-S264, 2005.

[57] S. B. Campbell, R. Walker, S. S. Tai, Q. Jiang, and G. R. Russ, "Randomized controlled trial of sirolimus for renal transplant recipients at high risk for nonmelanoma skin cancer," American Journal of Transplantation, vol. 12, no. 5, pp. 1146-1156, 2012.

[58] R. Robson, J. M. Cecka, G. Opelz, M. Budde, and S. Sacks, "Prospective registry-based observational cohort study of the long-term risk of malignancies in renal transplant patients treated with mycophenolate mofetil," American Journal of Transplantation, vol. 5, no. 12, pp. 2954-2960, 2005.

[59] J. O. O’Neill, L. B. Edwards, and D. O. Taylor, “Mycophenolate mofetil and risk of developing malignancy after orthotopic heart transplantation: analysis of the transplant registry of the International Society for Heart and Lung Transplantation," Journal of Heart and Lung Transplantation, vol. 25, no. 10, pp. 1186-1191, 2006.

[60] P. Braconnier, V. del Marmol, N. Broeders et al., "Combined introduction of anti-IL2 receptor antibodies, mycophenolic acid and tacrolimus: effect on malignancies after renal transplantation in a single-centre retrospective cohort study," Nephrology Dialysis Transplantation, vol. 27, no. 6, pp. 25472553, 2012 .

[61] J. R. Lake, K. M. David, B. J. Steffen, A. H. Chu, R. D. Gordon, and R. H. Wiesner, "Addition of MMF to dual immunosuppression does not increase the risk of malignant short-term death after liver transplantation," American Journal of Transplantation, vol. 5, no. 12, pp. 2961-2967, 2005.

[62] S. Vernino, D. R. Salomao, T. M. Habermann, and B. P. O’Neill, "Primary CNS lymphoma complicating treatment of myasthenia gravis with mycophenolate mofetil," Neurology, vol. 65, no. 4, pp. 639-641, 2005.

[63] N. Dasgupta, A. C. Gelber, F. Racke, and D. M. Fine, "Central nervous system lymphoma associated with mycophenolate mofetil in lupus nephritis," Lupus, vol. 14, no. 11, pp. 910-913, 2005.

[64] P. F. Finelli, K. Naik, J. A. DiGiuseppe, and A. Prasad, "Primary lymphoma of CNS, mycophenolate mofetil and lupus," Lupus, vol. 15, no. 12, pp. 886-888, 2006.

[65] B. P. O’Neill, S. Vernino, A. Dogan, and C. Giannini, "EBV-associated lymphoproliferative disorder of CNS associated with the use of mycophenolate mofetil," Neuro-Oncology, vol. 9, no. 3, pp. 364-369, 2007.

[66] G. Gebeyehu, V. E. Marquez, A. van Cott et al., "Ribavirin, tiazofurin, and selenazofurin: mononucleotides and nicotinamide 
adenine dinucleotide analogues. Synthesis, structure, and interactions with IMP dehydrogenase," Journal of Medicinal Chemistry, vol. 28, no. 1, pp. 99-105, 1985.

[67] E. Olah, Y. Natsumeda, T. Ikegami et al., "Induction of erythroid differentiation and modulation of gene expression by tiazofurin in K-562 leukemia cells," Proceedings of the National Academy of Sciences of the United States of America, vol. 85, no. 17, pp. 6533$6537,1988$.

[68] G. J. Tricot, H. N. Jayaram, E. Lapis et al., "Biochemically directed therapy of leukemia with tiazofurin, a selective blocker of inosine 5'-phosphate dehydrogenase activity," Cancer Research, vol. 49, no. 13, pp. 3696-3701, 1989.

[69] K. Malek, M. S. Boosalis, K. Waraska, B. S. Mitchell, and D. G. Wright, "Effects of the IMP-dehydrogenase inhibitor, Tiazofurin, in bcr-abl positive acute myelogenous leukemia-part I: in vivo studies," Leukemia Research, vol. 28, no. 11, pp. 1125-1136, 2004.

[70] N. Khanna, H. N. Jayaram, and N. Singh, "Benzamide riboside induced mitochondrial mediated apoptosis in human lung cancer H520 cells," Life Sciences, vol. 75, no. 2, pp. 179-190, 2004.

[71] D. Floryk and T. C. Thompson, "Antiproliferative effects of AVN944, a novel inosine 5-monophosphate dehydrogenase inhibitor, in prostate cancer cells," International Journal of Cancer, vol. 123, no. 10, pp. 2294-2302, 2008.

[72] J. M. Hamilton, M. W. Harding, T. Genna, and D. K. Bol, “A phase I dose-ranging study of the pharmacokinetics, pharmacodynamics, safety, and tolerability of AVN944, an IMPDH inhibitor, in healthy male volunteers," Journal of Clinical Pharmacology, vol. 49, no. 1, pp. 30-38, 2009.

[73] K. Ishitsuka, T. Hideshima, M. Hamasaki et al., "Novel inosine monophosphate dehydrogenase inhibitor VX-944 induces apoptosis in multiple myeloma cells primarily via caspaseindependent AIF/Endo G pathway," Oncogene, vol. 24, no. 38, pp. 5888-5896, 2005. 


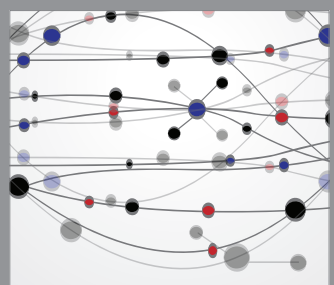

The Scientific World Journal
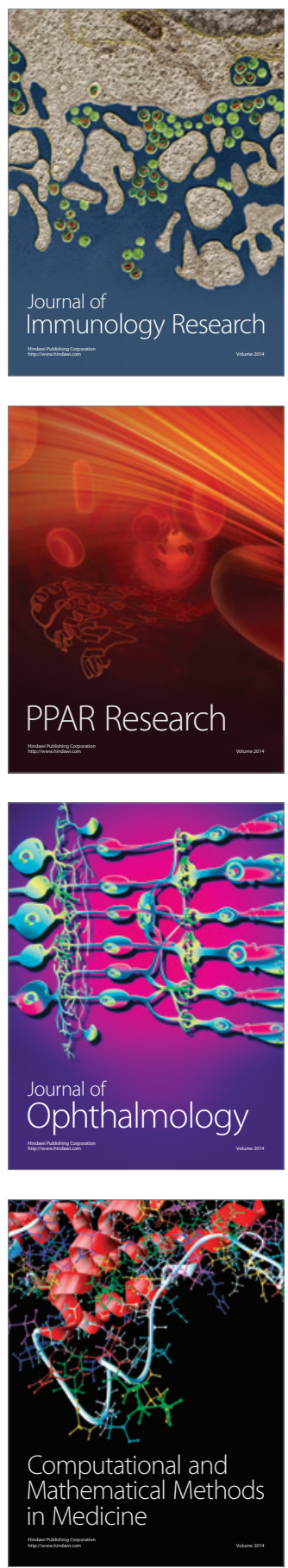

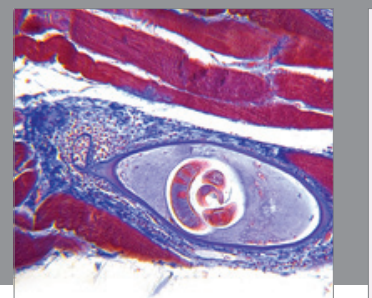

Gastroenterology

Research and Practice
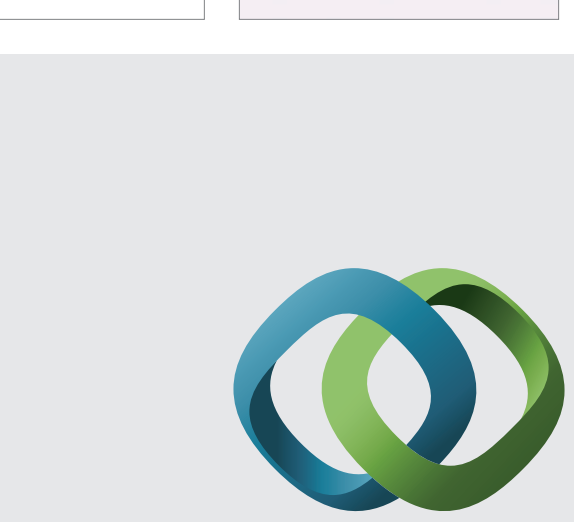

\section{Hindawi}

Submit your manuscripts at

http://www.hindawi.com
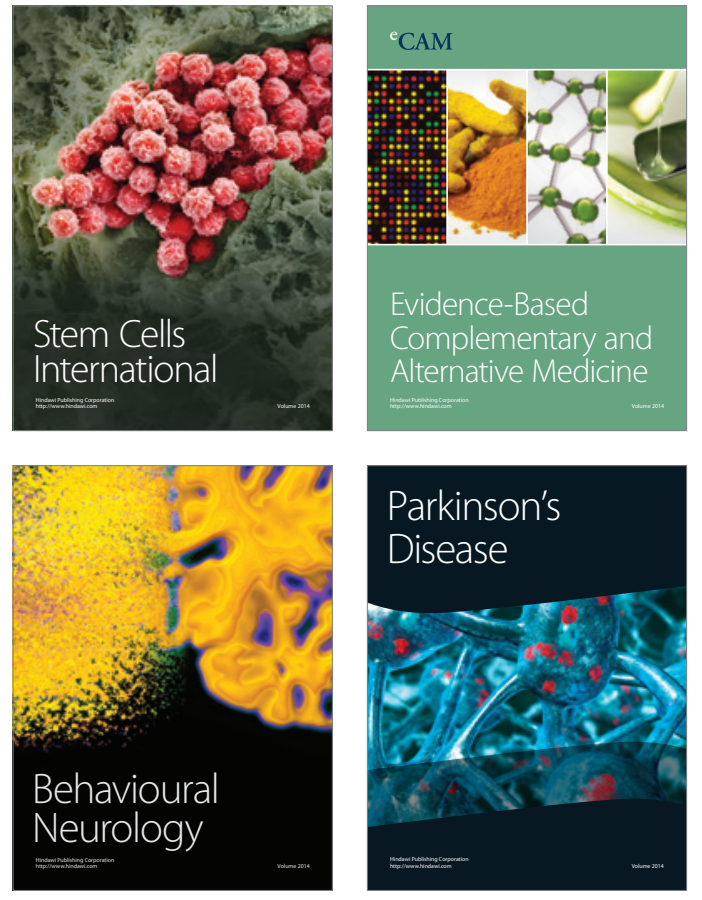
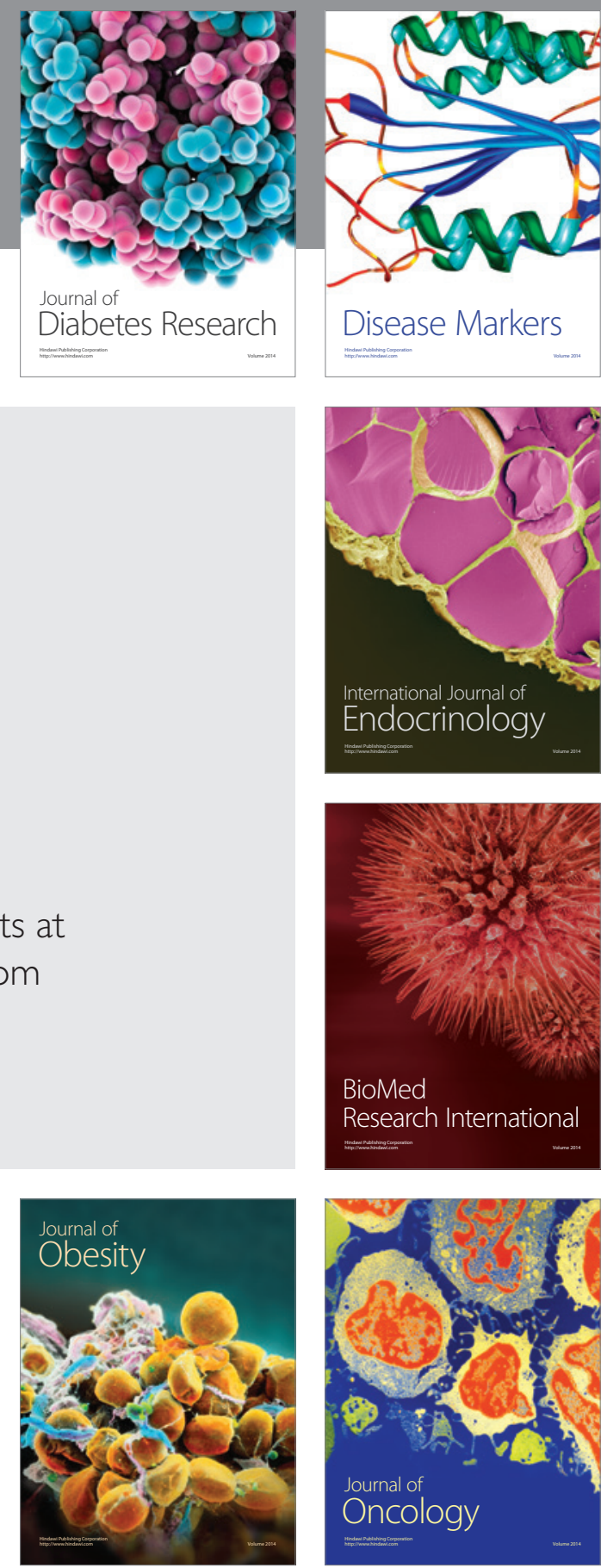

Disease Markers
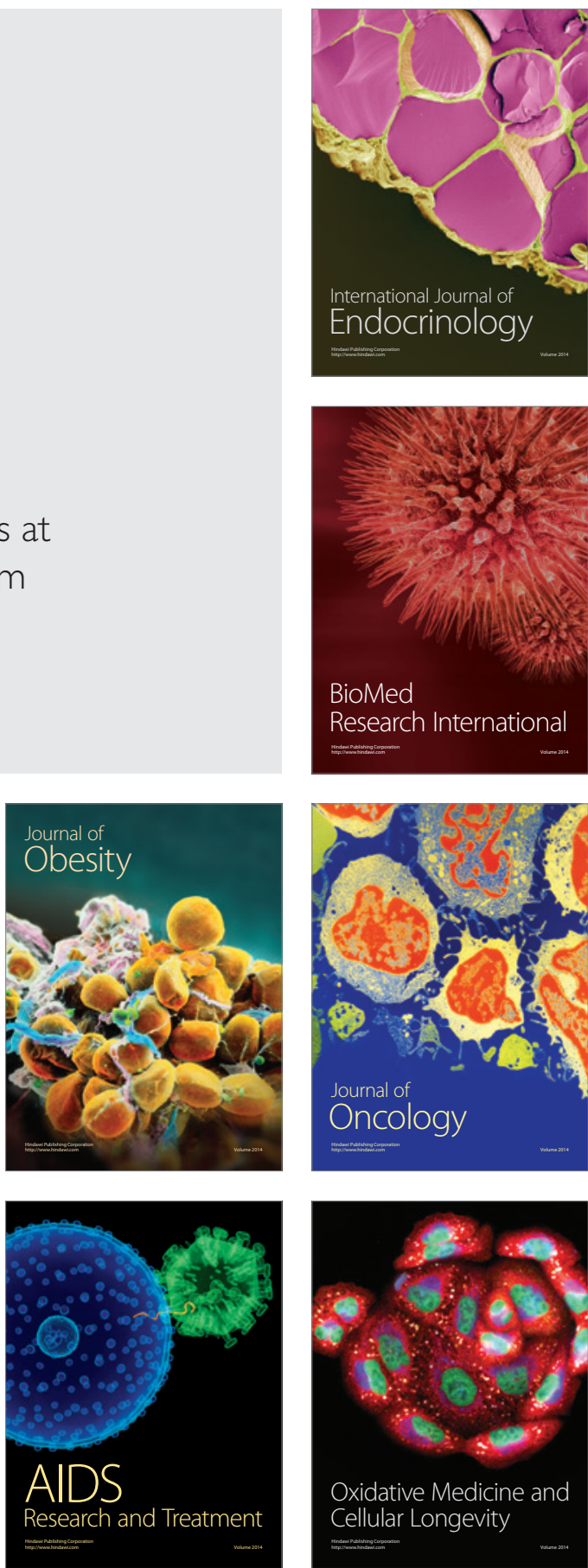